\title{
Relativistic self-compression approaching the Schwinger limit
}

\author{
PADMA K. SHUKLA, BENGT ELIASSON \\ and MATTIAS MARKLUND \\ Institut für Theoretische Physik IV, Ruhr-Universität Bochum, \\ D-44780 Bochum, Germany
}

(Received 26 April 2004)

\begin{abstract}
The effects of relativistic mass increase is considered in the context of intense laser-plasma interactions. It is found that the result of the relativistic effect is to enhance the self-compression and collapse of the intense laser pulse, making it possible to reach the Schwinger field limit, at which pair creation would need to be considered.
\end{abstract}

Recently, Bulanov et al. [1] discussed light intensification towards the Schwinger critical field limit caused by intense laser fields in plasmas. The purpose of the present paper is to show that, by using a simple analytical model, at high laser field intensities nonlinear effects associated with relativistic mass increase in intense laser fields and the local evacuation of electrons by relativistic laser ponderomotive force will produce compression (intensification) of a cylindrically symmetric laser pulse in plasmas. The evacuation of the plasma also lends support to the possibility of photon-photon scattering in laser-plasma systems.

The propagation of a circularly polarized intense laser pulse in an unmagnetized plasma is governed by $\partial_{t}^{2} \mathbf{A}-c^{2} \nabla^{2} \mathbf{A}+\omega_{\mathrm{p}}^{2} N \mathbf{A} / \gamma=0$ (see [2]), where $\mathbf{A}$ is the vector potential of the laser pulse, $\omega_{\mathrm{p}}=\left(4 \pi n_{0} e^{2} / m\right)^{1 / 2}$ is the unperturbed electron plasma frequency, $\gamma=\sqrt{1+e^{2}|\mathbf{A}|^{2} / m^{2} c^{4}}$ is the relativistic gamma factor including the electron mass variation in intense laser fields, $N=n_{\mathrm{e}} / n_{0}$ is the ratio of the electron number density to the background plasma number density $n_{0}, e$ is the magnitude of the electron charge, $m$ is the rest mass of the electrons, and $c$ is the speed of light in vacuum.

At intensities beyond $10^{18} \mathrm{~W} \mathrm{~cm}{ }^{-2}$, the electron quiver speed $v_{\mathrm{osc}}=6 \times 10^{-10} \mathrm{c \lambda} \sqrt{I}$ exceeds the speed of light, and hence nonlinear effects in plasmas cannot be ignored. Here $I$ is the intensity in $W \mathrm{~cm}^{-2}$ and $\lambda$ is the laser wavelength in microns. Thus, the relativistic ponderomotive force (see $[2,3]) \mathbf{F}=-m c^{2} \nabla \gamma$ of intense laser pulses will separate charges and thereby would create a huge ambipolar electric potential $\phi$ in the plasma. At equilibrium, the balance between the relativistic ponderomotive force and a slow electric force $e \nabla \phi$ will yield $\phi=\left(m c^{2} / e\right)(\gamma-1)$, which, when substituted into Poisson's equation, gives $N=1+\lambda_{\mathrm{e}}^{2} \nabla^{2} \gamma$. Here $\lambda_{\mathrm{e}}=c / \omega_{\mathrm{p}}$ is the electron skin depth, and the ions are assumed immobile. The electron density will be locally evacuated by the relativistic ponderomotive force of ultra-intense nonuniform laser fields. The laser pulse localization and compression would then occur 

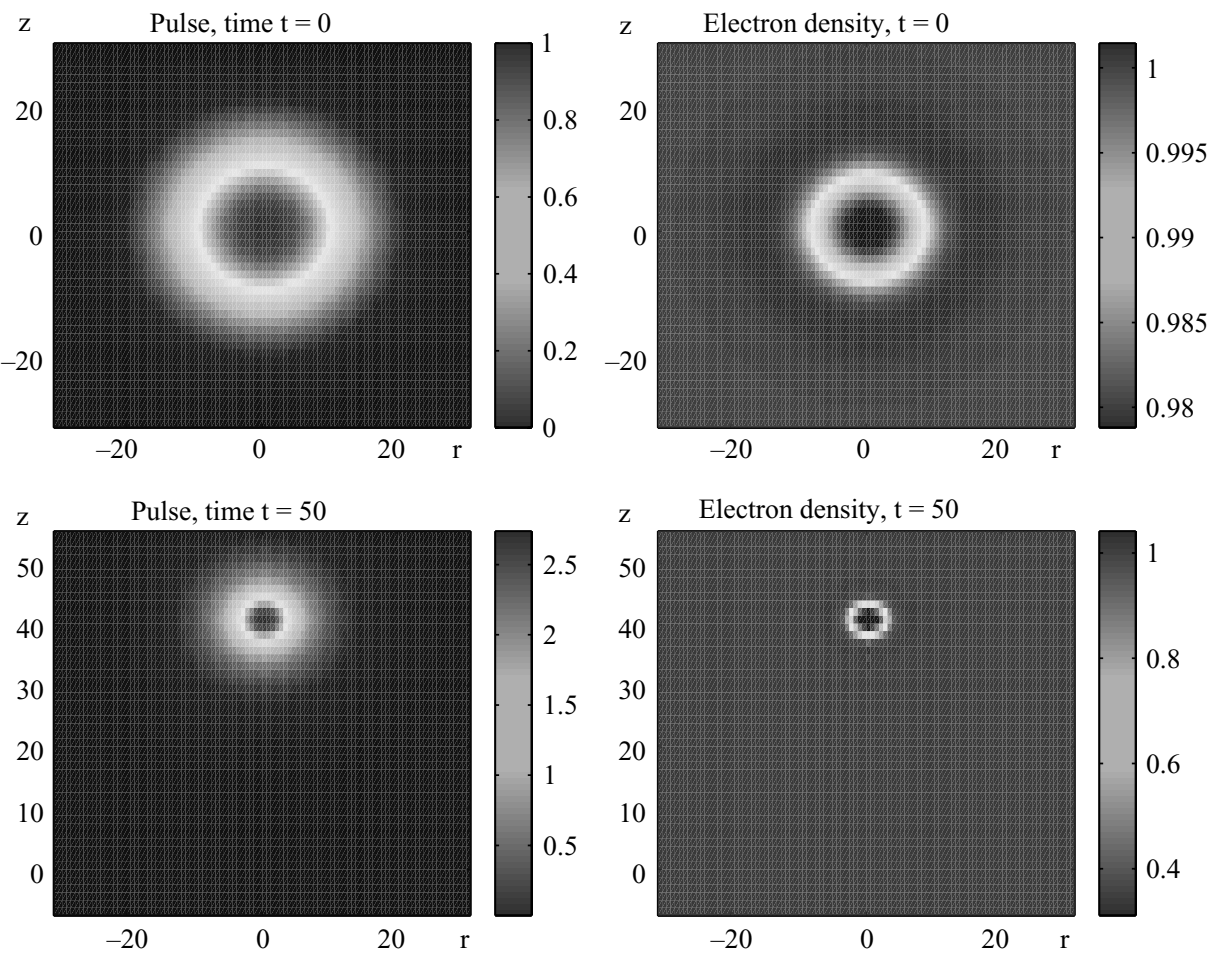

Figure 1. The variation of $I$ and $N$ against $r$ (the radial coordinate) and $z$ for an initial laser pulse that initially has a Gaussian shape. We observe the pulse compression and the formation of a light bullet.

due to nonlinearities associated with relativistic laser ponderomotive force created electron density evacuation and relativistic electron mass increase in the laser fields. This phenomena can be studied by means of the equation

$$
\frac{\partial^{2} \mathscr{A}}{\partial t^{2}}-\nabla^{2} \mathscr{A}+\frac{\mathscr{A}}{\sqrt{1+|\mathscr{A}|^{2}}}\left(1+\nabla^{2} \sqrt{1+|\mathscr{A}|^{2}}\right)=0,
$$

where $\mathscr{A}=e \mathbf{A} / m c^{2}$, and the time and space variables are in units of $\omega_{\mathrm{p}}^{-1}$ and $\lambda_{\mathrm{e}}$, respectively. For the propagation of a modulated laser pulse along the $z$-axis, from (1), after invoking the slowing varying envelope approximation, we obtain

$$
2 i \omega\left(\frac{\partial I}{\partial t}+v_{\mathrm{g}} \frac{\partial I}{\partial z}\right)+\nabla^{2} I+I-\frac{I}{P}\left(1+\nabla^{2} P\right)=0,
$$

where we have set $\mathscr{A}=(1 / 2) I(r, z, t)(\hat{\mathbf{x}}+i \hat{\mathbf{y}}) \exp (-i \omega t+i k z)+$ complex conjugate, and denoted $P=\left(1+I^{2}\right)^{1 / 2}$. Here the normalized laser frequency and the normalized laser group velocity are denoted by $\omega=\left(1+k^{2}\right)^{1 / 2}$ and $v_{\mathrm{g}}=k / \omega$, respectively. In the one-dimensional case (namely set $\nabla^{2}=\partial^{2} / \partial z^{2}$ in (2)), we have the localization of intense electromagnetic waves in the form of a large-amplitude one-dimensional bright soliton [2]. We have numerically solved (2) in order to study the evolution of a cylindrically symmetric modulated laser pulse. The results are displayed in Fig. 1. 
Initially, the pulse is assumed to have a Gaussian shape, $I=I_{0} \exp \left[-\left(z^{2}+r^{2}\right) /\right.$ 200], and we used the normalized wavenumber $k=2$ and initial pulse amplitude $I_{0}=1$. We note that the compression of the pulse envelope (left panels) is correlated with the excavation of the normalized electron density (right panels). Our numerical results reveal that self-compression of the pulse is more rapid when one accounts for the relativistic light ponderomotive force induced electron density depletion, in contrast to the constant density case (namely $N=1$ ). Physically, enhanced compression and self-focusing of an intense laser pulse occur due to the localization of light in a self-created electron density cavity.

The fact that evacuation of the plasma takes place, as the light intensification due to self-compression occurs, means that the situation discussed in [4], where the quantum electrodynamical effect of photon-photon scattering at high intensities takes place, could be realized in the next generation laser-plasma systems. Moreover, as the intensities in the evacuated region increase, the concept of vacuum catastrophic collapse, at which the pulse due to quantum vacuum nonlinearities self-compresses, may ensue [5]. The intensities that can be reached at this stage, in principle, surpass the Schwinger field, but then the process of pair creation has to be investigated and removed, since this would otherwise quickly dissipate the electromagnetic energy into Fermionic degrees of freedom.

In this paper, we have presented a model for the intensification of light in a plasma medium, accounting for the relativistic electron mass increase and the electron density expulsion by the relativistic ponderomotive force. The combined action of these two nonlinear effects gives rise to self-compression and collapse of laser pulses. It has been found that the pulse compression can yield field strengths reaching the Schwinger limit. Moreover, the evacuation of the plasma particles from the highintensity regions lends support to the notion of elastic photon-photon scattering in the next generation laser-plasma systems.

\section{Acknowledgements}

This research was partially supported by the European Commission through contract No. HPRN-CT-2001-00314 and by the Deutsche Forschungsgemeinschaft through the Sonderforschungsbereich 591 .

\section{References}

[1] Bulanov S. V. et al. 2003 Phys. Rev. Lett. 91, 085001.

[2] Yu, M. Y. et al. 1982 Phys. Fluids 25, 1049.

[3] Shukla, P. K. et al. 1986 Phys. Rep. 138, 1.

[4] Shen, B. and Yu, M. Y. 2003 Phys. Rev. E 68, 026501.

Shen, B., Yu, M. Y. and Wang, X. 2003 Phys. Plasmas 10, 4570.

[5] Marklund, M., Eliasson, B. and Shukla, P. K. 2004 JETP Lett. 79, 208. 